\title{
Preference for the interval schedule following multiple variable-ratio yoked-variable-interval schedule training
}

\author{
EUGENE L. EDMON and MICHAEL G. GRISHAM \\ University of Iowa, Iowa City, Iowa 52242
}

\begin{abstract}
Pigeons were studied on multiple variable-ratio yoked-variable-interval schedules in which components had equal rates of food reinforcement and appeared equally often on each of two keys. Interpolated between component changes on the final multiple schedule were 10-sec probes in which both schedule stimuli were present, one on each key. During multiple schedule training, variable-ratio response rates were greater than yoked-variable-interval rates; however, response rate differences in the components were not a function of the mean ratio value for the 40-to-320-ratio range studied. During the choice probes, subjects responded more to the stimulus associated with the interval schedule than to the one associated with the ratio schedule. It was concluded that pigeons prefer interval schedules over equal reinforcement rate ratio schedules, because the former generate fewer responses per reinforcement.
\end{abstract}

Bloomfield (1967) reported that two of three pigeons on multiple fixed-ratio variable-interval (FR VI) schedules required a higher FR than VI reinforcement rate for the absence of schedule interaction effects. Bloomfield argued that this result was consistent with other data (Appel, 1963), supporting the conclusion that FR schedules have aversive effects that subtract from the positive value of the reinforcer. He then speculated that these results might imply a general preference of pigeons for interval over ratio schedules.

Possible alternative interpretations of Bloomfield's results suggest that more direct evidence for a general preference would be of interest. For example, the VI preference in his data is consistent with the finding that aperiodic schedules are preferred over periodic schedules with the same reinforcement rate (Herrnstein, 1964; Killeen, 1968a). Also, Moore and Fantino (1975) have concluded that "pigeons prefer a second schedule to the extent that the response contingencies of the first schedule must be satisfied during discriminable periods of nonreinforcement" (p. 339). Since FR schedules require responding during discriminable periods of nonreinforcement, Moore and Fantino's analysis would imply a preference for the VI schedule in Bloomfield's results. The influence of either of these factors would limit the generality

This research was supported by funds from Biomedical Sciences Support Grant FR-07035 awarded to the University of lowa by the National Institute of Mental Health. The authors wish to thank Edward A. Wasserman, Gary Lucas, and James D. Deich for comments on the manuscript. Michael G. Grisham is now at Bell Laboratories, Holmdel, New Jersey. Requests for reprints should be sent to Eugene L. Edmon, Psychology Department, University of Iowa, Iowa City, Iowa 52242. of Bloomfield's conclusion about schedule preference per se. The present experiment investigated schedule preference with a method that would not allow these alternative explanations.

Equal reinforcement rates were produced for ratio and interval schedules by a within-subject yoking procedure whereby the interval schedule was based on the subject's performance on the ratio schedule. If this multiple variable-ratio yoked-variableinterval (VR y-VI) schedule produced the difference in ratio and interval response rates that is seen in the between-subject yoke (Ferster \& Skinner, 1957), then it would be clear that the subjects were discriminating the two schedules. A choice test between the component stimuli may then reveal any schedule preferences. Further, if a functional relation between VR value and discrimination performance could be found, the contribution of response rates to preference could be investigated. The search for this functional relation was a second purpose of the present experiment.

\section{Subjects \\ METHOD \\ Eight locally obtained homing pigeons were maintained at $80 \% \pm 15 \mathrm{~g}$ of their free-feeding weights. The subjects were given supplemental feeding of mixed grain after each daily session if necessary.}

\begin{abstract}
Apparatus
Only the side keys in four identical three-key pigeon chambers were used. The chamber interior was $29 \mathrm{~cm}$ long $\times 38 \mathrm{~cm}$ wide $\times 38 \mathrm{~cm}$ high. The reinforcer was $4-\mathrm{sec}$ access to mixed grain presented through a $5 \times 5.5 \mathrm{~cm}$ opening equidistant from the two $2.5-\mathrm{cm}$ side keys and $13 \mathrm{~cm}$ below them on the response panel. The only chamber illumination during reinforcement was from an ESB 24 lamp at $24 \mathrm{~V}$ in the BRS/LVE 114-10 grain hopper. Activation of the response switches required a force of .15-.20 N.
\end{abstract}


Industrial Electronics Engineers Series 10 readout projectors with No. 44 lamps at $5 \mathrm{~V}$ were mounted behind the translucent Plexiglas response keys and presented red or green colors. The houselight was a No. 44 lamp at $5 \mathrm{~V}$ in a housing that directed the light upward. Ventilation and masking noise were provided by an exhaust fan, and white noise was provided to a speaker in each chamber. Experimental control and data collection were managed from a separate room by a PDP-8/f computer (Digital Equipment Corporation) using the SKED software system.

\section{Procedure}

Keypecks were shaped by the method of successive approximations. Shaping was followed by 10 days of training to peck whichever key was illuminated with each peck reinforced and 42 reinforcers available each day. Red and green stimuli alternated with each response during this pretraining, and the right $(R)$ or left (L) key was lit by the appropriate color according to the repeating sequence RLRRLRLL. The sequence of VR values in the multiple VR $y$-VI schedules and the number of sessions each was in effect were: VR 80,28 ; VR 160, 48; VR 240, 28; VR 320, 24; VR 40,24 ; VR 80,36 . The VR 80 schedule was produced by repeated random sampling without replacement from the following set of values: $16,16,32,48,64,80,96,112,144,192$. The other VR schedules were produced by multiplying these values by an appropriate constant. Before being placed on the given multiple schedules, the eight pigeons had experience on between-subject VR y-VI schedules, four as masters and four as yokes.

For the multiple schedules, red or green stimuli appeared on the right or left keys in the same sequence used in pretraining. Thus, key color and key position were uncorrelated and the schedule discrimination could not be learned on the basis of position. Red was associated with the VR component for Pigeons 275 , 283,285 , and 287 , and with the $y$-VI component for the remaining pigeons. Scheduled component duration, including reinforcement time, was $90 \mathrm{sec}$, except with the VR 40 schedule, which had a component duration of $60 \mathrm{sec}$ because of computer memory limitations, which allowed yoking of at most 5 reinforcers per component.

The yoking was accomplished by beginning each session with the VR component of the multiple schedule. As the subject earned reinforcers on the VR schedule, the obtained interreinforcer intervals were stored by the computer. After the $90-\mathrm{sec}$ component duration, alternation to the y-VI component occurred. Reinforcers were then made available according to the interreinforcer intervals that had occurred during the just previous VR component. Each reinforcer made available on the yoked schedule was delivered following the next response or was cancelled by the availability of the next reinforcer. Uncollected reinforcers were also cancelled by component change. Component changes were separated by 5 -sec blackouts. Sessions were terminated after at least 40 reinforcers had been delivered and equal time had been spent in both components. Session duration (excluding hopper duration), the number of responses and reinforcements in each component, and the time between availability and delivery of each yoked reinforcer were recorded.

During the last six sessions on the final multiple VR $80 \mathrm{y}-\mathrm{VI}$ schedule, each component was followed by a 10 -sec presentation of both discriminative stimuli, one on each key. Stimulus position strictly alternated between left and right keys on successive choice trials. Thus, if red were on the right for a particular trial, it would be on the left during the next trial. Five-second blackouts preceded and followed the choice trials, and reinforcement never occurred during these trials. The number of responses to each stimulus was recorded.

\section{RESULTS}

Figure 1 presents response rates on the final 4-day block for each subject at each VR value. All subjects

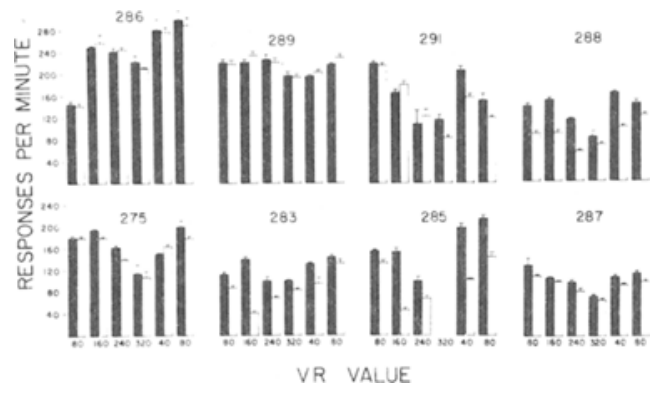

Figure 1. Average response rates for the final 4-day block on each multiple schedule for each pigeon. The solid bars represent VR response rates; one standard error above the mean is indicated at the top of each bar. Pigeon 285 ceased responding after 16 sessions on VR 320.

showed the same general tendency for both VR and VI absolute response rates to decline as the mean ratio value was increased. The VR value did affect performance then, but the degree of difference in VR and VI schedule response rates was found to be generally independent of the scheduled VR value. Four birds $(283,285,287$, and 288$)$ showed consistent VR, VI response rate differences at all VR values, two birds ( 275 and 291) discriminated on some of the schedules, and two birds (286 and 289) never discriminated.

The data on the final multiple VR $80 \mathrm{y}$-VI schedule presented in Figure 1 show that all subjects, except 286 and 289 , had a higher response rate on the VR schedule, indicated by a lack of overlap in the mean rates \pm 1 standard error. Table 1 presents relative response rates to the VI schedule for this final block and the overall choice proportions for each of the eight pigeons. During choice, all birds except 286 responded more to the stimulus associated with the y-VI schedule. Most importantly, all six pigeons that discriminated preferred the $y$-VI stimulus. The possibility that extent of preference was related to the difference in schedule response rates was assessed by calculating a Spearman $r$ coefficient between rank of VI relative rate (highest rank for lowest relative rate) and rank on relative frequency of choice responding to the VI stimulus. The ranks were determined before rounding the proportions. This test revealed no correlation between these variables $(r=.36, p>.10)$.

Subjects collected approximately $98 \%$ of all available $y-V I$ reinforcers. The average time between initial availability and delivery of these reinforcers was about $1 \mathrm{sec}$. The yoke was effective in maintaining equivalent rates and distributions of component reinforcers.

\section{DISCUSSION}

The results of this experiment provide direct support for Bloomfield's suggestion that pigeons prefer 
Table 1

Multiple VR 80 y-VI Schedule and Choice Relative Frequency of Responding to the VI Stimulus

\begin{tabular}{lcccccccc}
\hline & \multicolumn{8}{c}{ Subject } \\
\cline { 2 - 9 } & 275 & 283 & 285 & 286 & 287 & 288 & 289 & 291 \\
\hline Schedule & .47 & .48 & .40 & .49 & .47 & .46 & .52 & .44 \\
Choice & .65 & .66 & .60 & .44 & .65 & .67 & .62 & .73 \\
\hline
\end{tabular}

interval over ratio schedules, although further work is needed to establish the generality of this effect. Since the schedules employed here were of equal rate and distribution of reinforcement, the present results cannot be interpreted in terms of a preference based on periodicity vs. aperiodicity. Also, since periods of nonreinforcement should have been equally discriminable in the two components, Moore and Fantino's (1975) preference analysis would not seem to apply to these results either.

The general conclusion that ratio schedules produce higher response rates than interval schedules with equal reinforcement rates (Ferster \& Skinner, $1957)$ is supported by the present results. This outcome indicates that responses per reinforcement is lower on interval schedules, which may underlie the general preference for interval schedules. It is of interest that the present schedule and choice results, and the interpretation of those results just given, are in agreement with the recent model of schedule responding presented by Rachlin and Burkhard (1978). However, the role of responses per reinforcement in schedule preference is disputed.

Based on their results and other data (Killeen, 1968b; Neuringer, 1969), Moore and Fantino (1975) aruged that "a simple difference in rate of responding does not influence choice between two schedules" (p. 342). However, Arnett (1972) and Schuster (1969) have reported results consistent with the interpretation that responses per reinforcement does enter into schedule preference. Moore and Fantino discussed the possibility that Arnett's and Schuster's results were due to the stimulus manipulations employed to induce response rate differences in their two equal reinforcement-rate schedules. This limitation would not apply to the present results.

Although an eightfold range of VR values was investigated here, no functional relation between VR value and multiple schedule discrimination performance was discovered. This prevented the search for a functional relation between degree of preference and responses per reinfocement. Also, while a significant correlation between discrimination level and preference would have been of interest, little can be made of the failure to find such a correlation.

It was noted earlier that part of Bloomfield's preference argument was based on the conclusion that FR schedules were aversive to some degree. In turn, this conclusion was based on the demonstration that pigeons would respond to remove the stimulus associated with an FR schedule (Appel, 1963). Later work (Brown \& Flory, 1972) has shown that FI schedules also support escape responding at some interval values. There appear to be no reports of escape from either VR or VI schedules. This lack may be due to the belief that the fixed schedules support escape because they have a discriminable period of nonreinforcement. The results reported here suggest than an investigation of escape from VR would be of interest in that, if it occurred, it would offer an alternative to responses per reinforcement as a basis for the obtained interval schedule preference, assuming that escape from VI did not occur. Escape from VR might occur if response-based schedules are aversive.

\section{REFERENCES}

APPEL, J. B. Aversive aspects of a schedule of positive reinforcement. Journal of the Experimental Analysis of Behavior, 1963, 6, 423-428.

ARNETT, F. B. Inverse relation between choice and local response rate with a schedule of response-produced blackouts. Journal of the Experimental Analysis of Behavior, 1972, 17, 37-43.

Bloomfield, T. M. Behavioral contrast and relative reinforcement frequency in two multiple schedules. Journal of the EXperimental Analysis of Behavior, 1967, 10, 151-158.

Brown, T. G., \& Flory, R. K. Schedule-induced escape from fixed-interval reinforcement. Journal of the Experimental Analysis of Behavior, 1972, 17, 395-403.

Ferster, C. B., \& Skinner, B. F. Schedules of reinforcement. New York: Appleton-Century-Crofts, 1957.

Herrestein, R. J. Aperiodicity as a factor in choice. Journal of the Experimental Analysis of Behavior, 1964, 7, 179-182.

KiLlEEN, P. On the measurement of reinforcement frequency in the study of preference. Journal of the Experimental Analysis of Behavior, 1968, 11, 263-269. (a)

Killeen, P. Response rate as a factor in choice. Psychonomic Science, 1968, 12, 34. (b)

Moone, J., \& F antino, E. Choice and response contingencies. Journal of the Experimental Analysis of Behavior, 1975, 23, 339-347.

Neuringer, A. J. Delayed reinforcement versus reinforcement after a fixed-interval. Journal of the Experimental Analysis of Behavior, 1969, 23, 375-383.

Rachlin, H., \& Burkhard, B. The temporal triangle: Response substitution in instrumental conditioning. Psychological Review, $1978,85,22-47$.

SCHUSTER, R. H. A functional analysis of conditioned reinforcement. In D. P. Hendry (Ed.), Conditioned reinforcement. Homewood, Ill: Dorsey Press, 1969.

(Received for publication December 22, 1978; revision accepted March 7, 1979.) 